\title{
La intervención de terceros en el proceso civil: El interés como criterio delimitador de la calidad de tercero.
}

Sheila María Vilela Chinchay ${ }^{1}$

RESUMEN

Los terceros en el proceso civil constituyen una categoría que no ha resultado fácil de comprender. Existen dos concepciones de tercero que interesa anotar para analizar la intervención de terceros regulada en el C.P.C. Una vinculada al hecho de ser ajeno al proceso (sentido amplio) y otra vinculada a la ruptura del principio de dualidad del proceso (sentido estricto). Habrá quienes al intervenir en el proceso se conviertan en parte y como tal no es admisible su regulación dentro del acápite de la intervención de terceros, y habrá quienes al ingresar al proceso triangulen este y en consecuencia se constituyan en verdaderos terceros. Será determinante para identificar esta última categoría el tipo de interés con el que se solicita tutela al órgano jurisdiccional.

\section{PALABRAS CLAVE}

Terceros, partes, legitimidad, interés.

\section{SUMARIO}

1. Introducción 2. Consideraciones generales 3. El concepto de parte 4. La legitimidad como requisito para actuar en el proceso 5. El concepto de parte: El interés como criterio determinante 6. Conclusiones.

\footnotetext{
${ }^{1}$ Profesora de Derecho procesal civil y Coordinadora el área de derecho procesal y judicial de la Facultad de Derecho de la Universidad Católica Santo Toribio de Mogrovejo.
} 


\section{INTRODUCCIÓN:}

Los supuestos en los que sujetos distintos al demandante y al demandado participan en el proceso, son variados, así tenemos la intervención de testigos, la presentación de amicus curiae en los procesos constitucionales, la participación de los peritos informantes, la nueva y discutida posibilidad de actuar como partícipe (categoría reconocida por el Tribunal Constitucional) y los supuestos de intervención reguladas en los artículos 97 a 107.

Ser tercero puede significar ser un sujeto ajeno al proceso sin interés alguno en este, como sucede con el lector respecto de un proceso entre Juan y Ana sobre reivindicación de un bien. También podría significar, ser un sujeto que es ajeno al proceso entre Juan y Ana pero que goza de un interés en este y por último podría significar que se es tercero en tanto, estando al interior del proceso, el sujeto no encaja en la categoría de demandante o demandado, rompiendo así la dualidad del proceso. Para estos dos últimos casos, no nos queda duda de que el referido tercero será tal en tanto no ingrese al proceso, pero una vez dentro ¿se convertirá en parte? ¿Seguirá siendo tercero? o ¿asumirá una categoría distinta a la de parte y tercero?

Los supuestos de intervención de terceros regulados en el C.P.C. son nueve, a saber: Intervención coadyuvante, intervención litisconsorcial, intervención excluyente principal, intervención excluyente de propiedad o de derecho preferente, denuncia civil, aseguramiento de pretensión futura, llamamiento posesorio y llamamiento en caso de fraude o colusión. Analizadas estas figuras, advertimos que los supuestos de hecho están referidos a la participación no originaria de los sujetos, pero no se establece de modo claro cuál es el estatus con el que actuarán al interior del proceso, esto no ha animado a trabajar en el tema y determinar cuál es el factor de atribución de la calidad de tercero en el proceso.

A fin de lograr este objetivo, se ha estructurado el análisis partiendo de la regulación establecida en el C.P.C. en un segundo apartado se analiza el concepto de parte, en tanto su 
noción negativa se erige en el primer intento por esbozar el concepto de tercero, pasamos al tema de la legitimidad como exigencia para actuar en el proceso y terminamos el razonamiento, señalando cuál es el factor de atribución de la calidad de tercero en el proceso.

\section{CONSIDERACIONES GENERALES:}

La intervención de terceros es regulada en el C.P.C. en los artículos 97 a $107^{2}$. En la intervención litisconsorcial, la denuncia civil y el llamamiento posesorio, los sujetos que intervienen poseen una característica común, el tener un interés de tal naturaleza que al integrarse con posterioridad a la relación procesal, lo hacen en lugar de quien aparece demandado o al lado de cualquiera de las partes, con las mismas facultades que esta tiene. Situación parecida acontece en el supuesto de la intervención adhesiva, aunque con una restricción en las facultades. Por su parte la intervención excluyente principal, el llamamiento en caso de fraude y el aseguramiento de pretensión futura, pueden ser agrupadas considerando que el nuevo integrante del proceso no se coloca al lado de demandante y demandado ni tampoco en el lugar de estos ${ }^{3}$.

Definiremos, a partir de la norma, las características esenciales de cada una de estas instituciones. Así tenemos en el primer grupo:

“Artículo 98: Intervención litisconsorcial.- Quien se considere titular de una relación jurídica sustancial a la que presumiblemente deban extenderse los efectos de una sentencia, y que por tal razón estuviera legitimado para demandar o haber sido demandado en el proceso, puede intervenir como litisconsorte de una parte, con las mismas facultades de esta. Esta intervención puede ocurrir incluso durante el trámite en segunda instancia."

\footnotetext{
2 Intervención coadyuvante, intervención litisconsorcial, intervención excluyente principal, intervención excluyente de propiedad o de derecho preferente, denuncia civil, aseguramiento de pretensión futura, llamamiento posesorio y llamamiento en caso de fraude o colusión.

${ }^{3}$ No serán considerados en el análisis los supuestos de tercería preferente de pago y excluyente de propiedad por tratarse de una intervención especial que requiere de la actuación a través de la instauración de un proceso abreviado.
} 
- El sujeto que interviene se considera titular de la relación sustancial, en consecuencia será quien cuenta con la legitimidad para actuar en el proceso instaurado: Tiene legitimidad ordinaria.

- Como consecuencia lógica, posee las facultades de una parte.

"Artículo 102: Denuncia civil.- El demandado que considere que otra persona , además de él o en su lugar, tiene una obligación o responsabilidad en el derecho discutido, debe denunciarlo indicando su nombre y domicilio, a fin de que se le notifique del inicio del proceso"

- Otro sujeto es el responsable, otro ha de ser el sujeto pasivo de la acción.

- La responsabilidad puede ser compartida, son dos sujetos pasivos.

“Artículo 105: Llamamiento posesorio.- Quien teniendo un bien en nombre de otro, es demandado como poseedor de él, debe expresarlo en la contestación de la demanda...Si el citado comparece y reconoce que es el poseedor, reemplazará al demandado, quien quedará fuera del proceso... Si el citado no comparece o haciéndole niega su calidad de poseedor, el proceso continuará con el demandado, pero la sentencia surtirá efecto respecto de este y del poseedor por él designado...".

- El sujeto demandado inicialmente es un servidor de la posesión, en tanto conserva la posesión en nombre del poseedor. 4

- El sujeto pasivo de la acción ha de ser un poseedor y no el servidor de la posesión

- A través del llamamiento posesorio se busca la comparecencia del sujeto legitimado pasivamente.

En el segundo grupo tenemos:

Artículo 99: Intervención excluyente principal.- Quien pretenda, en todo o en parte, ser declarado titular del derecho discutido, puede intervenir formulando su exigencia

4 Artículo 897.- Servidor de la posesión: No es poseedor quien, encontrándose en relación de dependencia respecto a otro, conserva la posesión en nombre de este y en cumplimiento de órdenes e instrucciones suyas. 
contra demandante y demandado... El excluyente actuará como una parte más en el proceso..."

- Al parecer se rompe el principio de dualidad del proceso, esto porque el sujeto ingresa al proceso con una pretensión que se opone a demandante y demandado. Se da una triangulación del proceso como señala Monroy Gálvez 5 .

- Al señalar que actúa como una parte más, expresamente se reconoce tres partes en el proceso.

Artículo: Aseguramiento de pretensión futura.- La parte que considere tener derecho para exigir de un tercero una indemnización por el daño o perjuicio que pudiera causarle el resultado de un proceso, o derecho a repetir contra dicho tercero, lo que debiera pagar en ejecución de sentencia, puede solicitar el emplazamiento del tercero con el objeto de que en el mismo proceso, se resuelva además la pretensión que tuviera contra él.

- El demandado ingresa una pretensión adicional al proceso pero no contra el demandante sino contra un tercero, pretensión que no vincula al demandante.

Artículo 106: Llamamiento en caso de fraude o colusión.- Cuando en cualquier etapa del proceso se presuma fraude o colusión entre las partes, el juez, de oficio, ordenará la citación de las personas que puedan resultar perjudicadas, a fin de que hagan valer sus derechos..."

- El sujeto llamado a intervenir velará por sus derechos al interior del proceso sin que agregue pretensión alguna al proceso.

En el primer grupo de intervenciones, los sujetos autorizados para intervenir cuentan con legitimidad para actuar en el proceso, ya sea activa o pasiva, al lado de otro

5 MONROY GALVEZ, Juan, "Partes, acumulación, litisconsorcio, intervención de terceros y sucesión procesal en el Código Procesal Civil”, Estudios de Derecho Procesal, Ius et Veritas y Jurista Editores, Perú 2009 , pp. 355. 
sujeto o de modo individual. Recordemos que al constituirse la relación jurídica procesal ha de verificarse la concurrencia de unos requisitos esenciales como son las condiciones de la acción y los presupuestos procesales, entre los que encontramos a la legitimidad para obrar. La legitimidad ha de ser activa o pasiva y frente a los supuestos de falta o defecto en la legitimidad se procederá, si se trata de litisconsorcio necesario, del modo siguiente ${ }^{6}$ :

- El juez está facultado para integrar la relación procesal emplazando a la persona, si de la demanda o de la contestación es evidente que la decisión a recaer en el proceso le va a afectar, y en caso no tener la información necesaria para el emplazamiento devolverá la demanda y requerirá al demandante los datos para el emplazamiento al litisconsorte.

- Si el defecto se denuncia o es advertido por el juez con posterioridad a la notificación de la demanda, suspenderá el proceso hasta que se establezca válidamente la relación jurídica procesal.

Fuera del supuesto de litisconsorcio necesario, cuando se trata de la falta de legitimidad para obrar de un solo sujeto, se procede de la siguiente forma:

- En el supuesto de falta de legitimidad para obrar pasiva, se suspenderá el proceso hasta que se constituya válidamente la relación procesal. La denuncia del defecto se hará vía excepción.

- En el supuesto de falta de legitimidad para obrar activa, operará la improcedencia sin posibilidad de integración. ${ }^{7}$

El sujeto abstracto, que puede ser integrado en función de los artículos 95 y 451 puede ser integrado a través de los supuestos de intervención de terceros que se ha mencionado, lo que nos permite afirmar que en estos supuestos operará la conversión de tercero a parte.

\footnotetext{
${ }^{6}$ Ver artículos 95 y 451 del Código procesal civil.

${ }^{7}$ La no posibilidad de integración del sujeto activo en el proceso, se explica porque el derecho de acción, a diferencia del derecho de contradicción, sólo puede ser ejercitado de modo voluntario.
} 
En el segundo grupo de intervenciones, identificamos que se trata de sujetos que se integrarán (de modo forzoso o voluntario) al proceso y que tendrán respecto de demandante y demandado un interés distinto. Integrados al proceso, estos sujetos seguirían siendo llamados terceros pero ya no por ser ajenos al proceso, sino por romper con la estructura dual del proceso

A fin de determinar quién es tercero y quién es parte en el proceso, convendrá atender a los conceptos. La presencia de tercero en el proceso, como señala Gozaíni, sólo resulta doctrinariamente posible a partir de la llamada noción negativa de la condición de parte, es decir, resulta tercero quien no posee la investidura de parte, en razón de no ser demandante ni demandado. ${ }^{8}$ Será necesario, entonces atender a este concepto inicialmente.

\section{EL CONCEPTO DE PARTE:}

La calidad o condición de parte, dice Ortells Ramos, es un conjunto de situaciones jurídicas, de carácter activo y de carácter pasivo que, de acuerdo con la ley procesal, corresponden a las personas que se hallan respecto de un proceso de declaración en una posición determinada. ${ }^{9}$

Al respecto, tres cuestiones importantes, primero, se ha precisado qué implica ser parte, es decir, ser sujeto de determinadas situaciones jurídicas; segundo, se especifica cuál es el factor de atribución de la calidad de parte, esto es la ley procesal y tercero, parece quedar claro que el autor explica el concepto de parte desde la perspectiva de los $\underline{\text { efectos }}$ que siguen al hecho de que un sujeto sea considerado parte en el proceso. Ello hace necesario especificar cuál es la posición "determinada" (acaso otra situación jurídica, que ha de ser anterior a la descrita por el autor) en la que ha de encontrarse un sujeto para ser catalogado como parte y así goce del conjunto de situaciones jurídicas a que se refiere el autor.

\footnotetext{
${ }^{8}$ GOZAÍNI, Osvaldo A. "Elementos del Derecho Procesal Civil", Sociedad Anónima Editora, Comercial, Industrial y Financiera, Buenos Aires 2005, pp. 254.

9 ORTELLS RAMOS, Manuel y otros, "Derecho procesal Civil", 8ava. Edición, Editorial Thomson y Aranzadi, España, 2008, pp. 99.
} 
Quien es parte en un proceso goza de expectativas, posibilidades procesales, cargas, imperativos, deberes. Así, la veracidad, la probidad, la lealtad y la buena fe procesal son ejemplo de deberes de las partes; y la carga probatoria, la posibilidad de obtener una demanda fundada o una contestación de la demanda fundada, son ejemplo de carga y expectivas respectivamente.

Podrá apreciarse que estas situaciones jurídicas, poco o nada nos dicen sobre quién puede o no ser considerado parte en el proceso. Estas, constituyen el efecto de estar en una determinada posición (o de estar en una situación jurídica determinada) ${ }^{10}$.

Se da un paso hacia atrás y se advierte que una situación jurídica constituye el efecto de una causa llamada relación jurídica. El conjunto de situaciones jurídicas atribuidas a una parte en el proceso son efecto lógico de encontrarse al interior de la relación jurídica procesal. Esto constituye razón suficiente para concluir que si bien podría resultar válido atender a los efectos para definir el concepto de parte, ello no es suficiente por no establecer y delimitar qué es lo que le permite a un sujeto gozar de tal investidura.

\section{LA LEGITIMIDAD COMO REQUISITO PARA ACTUAR EN EL PROCESO:}

Qué es lo determinante en la atribución de la calidad de parte, es una cuestión que no ha sido considerada de modo expreso en la definición anotada en el apartado anterior, siendo esta trascendente para la comprensión del concepto. Ortells Ramos, se refiere a esta, aunque no en el concepto mismo, y señala que para la atribución de la calidad de parte basta con que el sujeto realice un específico acto procesal, el acto de interponer la demanda, designarse como demandante y designar en ella al demandado; estableciendo, desde el principio quién ostenta tal calidad a fin de que sean citados a juicio. ${ }^{11}$ Esto

\footnotetext{
${ }^{10}$ Importa en ese sentido, atender a la definición de situación jurídica. Messineo, citado por Torres Vásquez la define como el conjunto de efectos que se derivan de una relación entre personas, en tal sentido, esta definición incluiría en sí misma la noción de relación jurídica y, en cierto modo, coincidiría con ella; de igual forma a veces la situación jurídica asume el perfil del status, consistente en una cualidad jurídica del sujeto de la cual se derivan derechos y deberes. TORRES VASQUEZ, Anibal, "Introducción al Derecho: Teoría General del Derecho", Tercera edición, Idemsa, Perú 2006. pp. 407.

${ }^{11}$ ORTELLS RAMOS, Manuel y otros, Ob, cit. pp. 100.
} 
complementa el razonamiento en tanto se pone la atención en el acto de inicio de la relación procesal.

Por su parte Enrique Falcón, citado por Hinostroza Mingues, apuesta por un concepto en el que atiende a la actuación del sujeto frente al órgano jurisdiccional pero incluye en el concepto a todo aquel que solicita tutela, siendo para este indiferente el tipo de tutela reclamada; en este sentido para Falcón será parte todo aquel que reclama el auxilio de la jurisdicción en un proceso, en forma originaria, sucesiva, principal, incidental o accesoria, y los que intervienen como consecuencia de tal reclamo. ${ }^{12}$

Rosemberg, parte de la afirmación del derecho material como criterio delimitador del concepto: "No se es parte en el proceso civil como titular de la relación jurídica controvertida, sino actor es quien afirma el derecho (material), y demandado aquel contra el que se lo hace valer. Para la posición de parte procesal no tiene importancia si el actor es el poseedor del derecho y si el demandado es el verdadero obligado o afectado. Muchas veces de acuerdo con el derecho material, están facultados para la gestión procesal y son partes personas distintas de los portadores del derecho o de la relación jurídica controvertida.... Es una cuestión distinta, si la parte es la verdadera, es decir si el derecho existe y corresponde precisamente al actor frente al demandado, y si las partes tienen la capacidad de llevar el proceso. Esta cuestión resuelve sobre el éxito de la demanda y determina el contenido de la sentencia, pero no determina quién es parte "13.

Para Satta, la parte no puede disociarse de la titularidad del interés que se hace valer en juicio, en definitiva no puede desatender a la titularidad de la acción; pero en tanto la acción se concreta en la demanda con la postulación del juicio como favorable, es la demanda la que atribuye la calidad de parte, de donde resulta exacta la fórmula tradicional que define a la parte como el sujeto que en su propio nombre demanda o el sujeto contra quien la demanda, siempre en nombre propio, es propuesta. ${ }^{14}$

\footnotetext{
${ }^{12}$ HINOSTROZA MINGUEZ, Alberto, “Sujetos del proceso civil”, Primera Edición, Gaceta Jurídica, Perú 2004. pp. 203.

${ }^{13}$ Ibidem,. pp. 205.

${ }^{14}$ Ibidem, pp. 216.
} 
Con algunos matices, no poco importantes, estos autores coinciden en señalar en que es la realización de un determinado acto procesal lo que determina la calidad de parte: La actuación de un sujeto en contra de otro a través del órgano jurisdiccional, entendiéndose por tal el inicio del proceso judicial a través de la demanda en la que queda materializado el derecho de acción. Compartimos esta afirmación en cuanto a que es la actuación de un sujeto en contra de otro lo que pone en evidencia a las partes en el proceso, sin embargo no parece acertado establecer como único momento de actuación la interposición de la demanda.

En el primer apartado señalábamos cómo es posible que la relación procesal sea integrada de oficio o por pedido de una de las partes, desde esa perspectiva es perfectamente correcto afirmar que un sujeto será parte en el proceso aun cuando no aparezca determinado en la demanda. Es necesario considerar el interés con el que intervienen.

Precisamente, la discrepancia en las posturas de los autores citados, la encontramos en la referencia al tipo de interés con el que se acude al órgano jurisdiccional. La referencia a este interés ha llevado a diversos autores a considerar las categorías intermedias: partes principales, accesorias, incidentales o subordinadas. Al órgano jurisdiccional se puede acudir con pedidos distintos, el derecho a la tutela jurisdiccional efectiva no se reduce al acto de interposición de demanda y de contestación de la misma; por ejemplo, no puede desconocerse que un pedido de desafectación de un bien embargado a un sujeto que no es el obligado en un proceso, se constituye en una forma de pedir tutela jurisdiccional al Estado. El sujeto que pide la desafectación cuenta con una cualidad especial, que en derecho procesal se denomina legitimidad, concepto que es transversal en el proceso, de tal manera que podemos hablar de la legitimidad del demandante, del demandado, del tercero o de la legitimidad para plantear nulidades.

Tenemos así una legitimidad que varía de contenido atendiendo a un interés específico. 
La legitimidad para ser parte ha de ser entendida como la identidad lógica que existe entre demandante o demandado y aquella persona a la que en abstracto la ley le autoriza a actuar como tal ${ }^{15}$, luego, cabría una inicial afirmación: Sólo quien está legitimado para accionar o contradecir, puede, al mismo tiempo, en que se instaura el juicio con la constitución de relación jurídica procesal, asumir la calidad de parte, esto es, de actor o de demandado en juicio. Sin embargo, ello no es así, pensemos en un proceso iniciado por un sujeto (demandante) que carece de legitimidad para obrar y dicha carencia es advertida en la etapa de saneamiento procesal, ya sea de oficio o a instancia de parte (vía excepción), a nadie se le podría ocurrir pensar que aquel sujeto nunca fue parte en el proceso porque carecía de la cualidad legitimadora y que el proceso nunca existió. Presentada la demanda, el órgano jurisdiccional en cumplimiento de su deber de administrar justicia, actúa y trata a aquel sujeto como parte y en razón de ello examina si puede pronunciarse o no sobre el fondo de la litis, esto quiere decir que lo trató como parte y le exigió las cualidades requeridas por la norma procesal para el caso concreto.

Ha de tenerse en cuenta que se trata sólo de una declaración de calidad, esto no puede ser perdido de vista. Ello puede explicarse en tanto siempre quien demande será aquel que considere que un bien le es debido aun cuando no le sea debido y le interesará que quien debe responder sea quien actúe en el proceso en calidad de demandado y de no ser así, como sucede en los supuestos de error o de fraude, quien a su conveniencia o creencia deba actuar en calidad de demandado. Hecha la declaración, se les tratará como tales para analizar su legitimidad, y de no contar con ésta, simplemente operará la improcedencia o la integración del o los sujetos legitimados.

\footnotetext{
${ }^{15} \mathrm{La}$ legitimidad es una cualidad o condición de las partes en relación con concretos procesos o, lo que es igual, con los concretos objetos de unos procesos. En DE LA OLIVA SANTOS, Andrés y DIEZ - PICAZO JIMENEZ, Ignacio, "El proceso de declaración", Segunda. Edición, Editorial Centro de Estudios Ramón Areces S.A. España, 2001, pp. 125 - 126.
} 


\section{EL CONCEPTO DE TERCERO: EL INTERÉS COMO CRITERIO DETERMINANTE}

Dos son las perspectivas que nos permitirían entender el tema de los terceros. Primero, es tercero aquel que es ajeno a un proceso, el mismo que podrá gozar o no de interés; segundo, es tercero aquel que está dentro del proceso pero no se coloca ni en lugar, ni al lado de demandante o demandado. Esto último puede esbozarse de la siguiente manera: Atendiendo al principio de dualidad del proceso, será tercero quien no encaje en la estructura dual del proceso y en tanto tal no le corresponderá las situaciones jurídicas de quien ostenta la calidad de parte ${ }^{16}$.

En palabras de Ramiro Podetti, “... los sujetos clásicos son dos: actor: Primus y demandado Secundus..." y aquel que no es primus ni secundus en tanto sujetos originarios (designados en la demanda) será Tertius. ${ }^{17}$ será tertius porque no es originario, esto es, se trata de un nuevo sujeto que además es distinto de primus y secundus aunque sea sólo en matices de su interés.

Son dos los criterios que sirven para definir al tercero, según Podetti, primero, el no ser originario y segundo, el tipo de interés. De estos dos criterios se considera relevante el segundo.

Sobre el primer criterio, insistimos en que la determinación a través de la demanda de quiénes serán las partes en el proceso no restringe la atribución de tal calidad a quienes actúan con posterioridad a la interposición de la misma. En todo caso convendría asumir lo señalado por Gozaíni, se es tercero mientras no se tenga la calidad de parte, pues una vez adquirida, la condición para actuar se confunde con los supuestos de litisconsorcio, vale decir, se pierde la condición de tercero para ser parte adherente a uno de los litigantes ${ }^{18}$. O como lo señala Ortells Ramos, el tercero puede dejar de serlo y adquirir la condición de

\footnotetext{
${ }^{16}$ Según el diccionario de la real academia de la lengua, tercero es aquella "persona que no es ninguna de dos o más de quienes se trata o que intervienen en un negocio de cualquier género".

${ }^{17}$ PODETTI, J. Ramiro, “Tratado de la Tercería”, Tercera edición, EDIAR S.A., Buenos Aires 2004, pp. 37.

${ }^{18}$ GOZAÍNI, Osvaldo A. Ob, cit. pp. 254.
} 
parte procesal si la afectación que le produce el proceso pendiente es suficiente para colocarle en una de las posiciones legitimantes previstas por la ley ${ }^{19}$, esto nos lleva a hablar del segundo criterio.

El proceso es un todo que comprende un elemento de importancia única, el ser humano, quien unido a un conjunto de hechos y situaciones particulares hacen del proceso una institución compleja y además de cambio constante dada la condición histórica del ser humano. Frente a esta constatación, con el devenir de los años se ha ido observando cómo surgen situaciones de hecho no previstas por la norma y que luego urge regular. Dentro de estas situaciones no previstas, en algún punto aparecen intereses de personas que de acuerdo a la relación material no tendrían razón para formar parte del proceso, no obstante, ver perjudicados sus intereses. Luego, se buscó la forma de integrarlos y surge, como anota Montero Aroca, el concepto de legitimidad, "En su origen el concepto de legitimación no nace para explicar los supuestos en que los titulares de una relación jurídica material, se convierten en partes del proceso, sino que por medio de él se pretende dar sentido a aquellos otros supuestos en los que las leyes permiten que quien no es sujeto de una relación jurídica material se convierta en parte del proceso... „20

El mismo razonamiento se aplica, para el supuesto de los intereses que si bien no generan legitimidad extraordinaria ${ }^{21}$, sí generan un tipo de legitimidad que permite a determinados sujetos intervenir en el proceso, sujetos a los que se les ha denominado tercero. $^{22}$

\footnotetext{
${ }^{19}$ ORTELLS RAMOS, Manuel, Ob, cit. pp. 110.

${ }^{20}$ MONTERO AROCA, Juan y FLORS MATÍES, José, “Tratado de juicio verbal”, Parte primera, Editorial Thomson y Aranzadi, España, 2004, pp. 328.
${ }^{21}$ Montero Aroca sí considera a la legitimidad con que cuentan los terceros como un supuesto de legitimidad extraordinaria. Esta afirmación se comprende si sólo hablamos de la intervención coadyuvante, pero no de los restantes tipos de intervención. Ver MONTERO AROCA, Juan, "Legitimidad para obrar y derecho jurisdiccional”, en Estudios de Derecho Procesal Civil, Ius et Veritas y Jurista Editores, Perú 2009, pp. 114.
${ }^{22}$ La legitimación permite diferenciar dos figuras: partes principales y partes subordinadas, así, las partes principales: "Son partes principales, quienes por ser titulares de la relación jurídica material debatida y por verse expuestos a los futuros efectos directos de la sentencia, están legitimadas para deducir la pretensión u oponerse a ellas, delimitan el objeto del proceso y generan la obligación del juez de congruencia en la sentencia, contra la que pueden recurrir con independencia; si dicha relación jurídica es además disponible, podrán provocar la finalización anormal del proceso a través de los actos de disposición de la pretensión (desistimiento, renuncia, allanamiento, etc).Las partes subordinadas (los intervinientes adhesivos o


Según el interés que se tenga la legitimidad ganará o perderá fuerza y ello determinará las facultades que se le deban conceder. Las posibilidades son las siguientes:

- Si los sujetos no ingresan pretensión alguna al proceso y se ubican al lado o en lugar de demandante o demandado, les corresponderá actuar como partes. La calidad de tercero se pierde en el momento en que ingresan al proceso.

- Si los sujetos ingresan o discuten una pretensión distinta o velan por un interés distinto, ubicándose contra demandante y demandado estaremos ante un tercero en sentido estricto, este es el supuesto de hecho acogido en los artículos 99, 104 y 106 del CP.C. (Intervención excluyente principal, aseguramiento de pretensión futura y llamamiento en caso de fraude o colusión).

Hecho este análisis, podemos afirmar que es el interés declarado por los terceros (en sentido amplio) lo que determinará su calidad al interior del proceso.

\section{CONCLUSIONES:}

Para ser considerado parte se necesita de la declaración a la que se refiere Ortells Ramos, pero esta declaración que puede ser posterior a la interposición de la demanda tiene un sustento, así tal declaración depende de considerarse con un interés jurídicamente tutelado y no meramente subjetivo claro está, ello con independencia de si efectivamente goza de este interés o no. Se desplaza el factor de atribución de la calidad de parte hacia el interés declarado por el sujeto que intervendrá en el proceso.

Todo sujeto que se encuentra fuera del proceso será considerado tercero en sentido amplio. Presentada su solicitud de ingreso al proceso, dependerá del interés declarado

coadyuvantes), se encuentran en una relación jurídica dependiente de otra principal, que es la que se debate en el proceso y de la que son titulares las partes principales, por lo que tan sólo han de sufrir los efectos reflejos o indirectos de la sentencia; por ello ni delimitan el objeto del proceso, ni pueden transigir sobre el mismo, ni pueden impugnar la sentencia con independencia. Simplemente coadyuvan con sus actos de postulación al éxito de la pretensión o defensa de la parte principal. "En GIMENO SENDRA, Vicente, "Derecho procesal Civil: I El proceso de declaración, parte general", Segunda Edición, Editorial COLEX, Madrid 2007, pp. 100. 
que sustenta su legitimidad lo que determinará las características de su actuación al interior del proceso. Podrá convertirse en parte o triangulará la relación procesal. $\checkmark$

En los supuestos de intervención litisconsorcial, denuncia civil y llamamiento posesorio, el sujeto dejará de ser tercero al ingresar al proceso.

En los supuestos de intervención excluyente principal, aseguramiento de pretensión futura y llamamiento en caso de fraude o colusión, el sujeto que interviene será tercero en sentido estricto en tanto rompe con el principio de dualidad del proceso. 